\section{Drs. Mavragani and Moutsopoulos reply}

To the Editor:

We were delighted to read the letter by Killian, et al, which challenges the use of the terms primary and secondary human Sjögren syndrome (SS) even in experimental animal models of $\mathrm{SS}^{1}$. These terms were coined almost 40 years ago on the basis of a study that revealed clinical, immunologic, and genetic differences among patients expressing sicca manifestations alone and patients with rheumatoid arthritis (RA) and SS. Despite the lack of appropriate studies, the rheumatology community expanded the term secondary SS to include all patients with autoimmune disorders who expressed sicca manifestations. Of note, subsequent reports on patients with systemic lupus erythematosus (SLE) or scleroderma and sicca features did not confirm observations similar to those seen in RA with sicca complaints ${ }^{2}$.

The MRL/MpJ mouse is an autoimmune strain that develops lacrimal and salivary gland inflammation (dacryoadenitis and sialadenitis) and has been long considered a model for human SS. Two main substrains of $\mathrm{MRL} / \mathrm{MpJ}$ mice are recognized: the MRL/MpJ-Fas+/Fas+ (MRL/+) and the MRL/MpJ-Faslpr/Faslpr (MRL/lpr). In the latter model, defective Fas mediated apoptosis of lymphocytes in peripheral lymphoid organs occurs as a result of a Fas ligand mutation, and systemic autoimmune disease develops with a wide range of manifestations [e.g., arthritis, glomerulonephritis, vasculitis central nervous system (CNS)/peripheral NS (PNS) inflammation, or massive lymphadenopathy]. In addition to dacryoadenitis and sialadenitis, both strains developed antinuclear antibodies, with the MRL/lpr mice displaying also autoantibodies against Ro/SSA and La/SSB autoantigens ${ }^{3,4}$.

With this in mind, SS experimental animal models sharing features related to both glandular (diminished salivary and lacrimal secretions) and extraglandular involvement reminiscent of those seen in SLE (e.g., glomerulonephritis, CNS/PNS inflammation) could be erroneously classified as models of "secondary" SS and therefore characterized as "inadequate" for studies investigating the potential efficacy of novel targeted therapies for primary $\mathrm{SS}^{1}$. However, it should be emphasized that these immunological treatment approaches are indeed expected to be effective in SS patients with systemic extraglandular manifestations and excessive immune activation rather than those with local inflammation confined to salivary glands. Moreover, it is worth mentioning that though glomerular involvement is considered mainly an SLE-related rather than an SS-related feature, earlier and more recent works documented the presence of membranoproliferative and membranous glomerulonephritis in patients with "primary" $\mathrm{SS}^{5,6}$. These patients were characterized by the presence of mixed monoclonal cryoglobulins and kidney immunopathology revealing predominant tissue deposition of $\operatorname{IgM}^{5}$. Peripheral neuropathy is another well-known manifestation of primary $\mathrm{SS}^{7}$, which together with glomerulonephritis, lymphadenopathy, and autoantibodies against Ro/SSA and La/SSB autoantigens, among other manifestations, has been shown to be highly predictive for lymphoma development in these patients ${ }^{8,9}$. The MRL/MpJ experimental animal model is an excellent model for the evaluation of therapeutic modalities because in addition to sicca manifestations, the model expresses extensive lymphopro- liferation affecting parenchymal organs ${ }^{3}$. Unfortunately, at present, management of SS is mainly limited to secretagogues and excretion replacement, while immunosuppressive agents are efficacious neither for alleviation of SS features nor for the prevention of disease progression ${ }^{10}$.

CLIO P. MAVRAGANI, MD, Department of Physiology, School of Medicine, National and Kapodistrian University of Athens; HARALAMPOS M. MOUTSOPOULOS, MD, FRCP, FACP, Athens Academy, Chair Medical Sciences/Immunology, Athens, Greece. Address correspondence to Dr. C.P. Mavragani, Department of Physiology, School of Medicine, National and Kapodistrian University of Athens, M. Asias 75, 11527, Athens, Greece. E-mail: kmauragan@med.uoa.gr

\section{REFERENCES}

1. Killian M, Batteux F, Paul S. The MRL/lpr mouse model: an important animal model for systemic Sjögren syndrome and polyautoimmunity. J Rheumatol 2020;47:157.

2. Mavragani CP, Moutsopoulos HM. Primary versus secondary Sjögren syndrome: is it time to reconsider these terms? J Rheumatol 2019;46:665-6.

3. Watanabe-Fukunaga R, Brannan CI, Copeland NG, Jenkins NA, Nagata S. Lymphoproliferation disorder in mice explained by defects in Fas antigen that mediates apoptosis. Nature 1992;356:314-7.

4. Lavoie TN, Lee BH, Nguyen CQ. Current concepts: mouse models of Sjögren's syndrome. J Biomed Biotechnol 2011;2011:549107.

5. Moutsopoulos HM, Balow JE, Lawley TJ, Stahl NI, Antonovych TT, Chused TM. Immune complex glomerulonephritis in sicca syndrome. Am J Med 1978;64:955-60.

6. Goules AV, Tatouli IP, Moutsopoulos HM, Tzioufas AG. Clinically significant renal involvement in primary Sjögren's syndrome: clinical presentation and outcome. Arthritis Rheum 2013; 65:2945-53.

7. Pavlakis PP, Alexopoulos H, Kosmidis ML, Mamali I, Moutsopoulos HM, Tzioufas AG, et al. Peripheral neuropathies in Sjögren's syndrome: a critical update on clinical features and pathogenetic mechanisms. J Autoimmun 2012;39:27-33.

8. Skopouli FN, Dafni U, Ioannidis JP, Moutsopoulos HM. Clinical evolution, and morbidity and mortality of primary Sjögren's syndrome. Semin Arthritis Rheum 2000;29:296-304.

9. Fragkioudaki S, Mavragani CP, Moutsopoulos HM. Predicting the risk for lymphoma development in Sjogren syndrome: an easy tool for clinical use. Medicine 2016;95:e3766.

10. Mavragani CP, Moutsopoulos HM. Conventional therapy of Sjogren's syndrome. Clin Rev Allergy Immunol 2007;32:284-91.

First Release September 15 2019; J Rheumatol 2020;47:1; doi: $10.3899 /$ jrheum.190837 